\title{
IMPROVEMENTS IN WATER INTERVENTION PROJECTS THROUGH PRODUCT DESIGN METHODS
}

\author{
Richard HARLOW and Lyndon BUCK \\ Buckinghamshire New University
}

\begin{abstract}
At a 2018 community discussion workshop for the EU Horizon 2020 funded WATERSPOUTT project in Chikwawa, Malawi, village representatives discussed water collection from the Shire river. WATERSPOUTT aims to provide safe drinking water to sub-Saharan African communities who rely on unsafe sources. A consortium of EU and African researchers is carrying out a technological development programme to advance applications based on Solar Disinfection (SoDis), providing clean potable water. The water in Chikwawa is polluted by sewage from upstream, bodies that have fallen in due to accidents and dirt and bacteria stirred up by rain, increasing the incidence of illness. Where products have been successful in previous projects, the user is limited to a specific process and when their situations change the technologies are no longer suitable. The constraints of these projects raise issues vital for consideration by design students such as localised production, sustainability, co-design and cultural appropriation. Design is a tool for producing products to solve a user's daily problems with the potential to develop long lasting solutions for those not in a position to create them for themselves. This paper reviews past interventions where design methods have been implemented, their effects, and primary research from this project. Discussing the importance of user engagement and the involvement of cultural factors in the advancement of new product development, the paper provides new considerations in user centred design for students and educators while discussing how design methods could be used to improve long term sustainability of intervention product design.
\end{abstract}

Keywords: Product design, water treatment, solar disinfection, filtration, sustainability

\section{INTRODUCTION}

"The main trouble with design schools seems to be that they teach too much design and not enough about the ecological, social, economic, and political environment in which design takes place." [1]. According to the World Health Organisation (WHO) [2] 844 million people globally do not have access to safely managed drinking water services, with 159 million dependent on surface water sources. The WATERSPOUTT project has worked to provide solar disinfection (SoDis) resources for the disinfection of local water for target communities in Ethiopia and Malawi.

This paper outlines past aid and intervention projects highlighting the opportunities and barriers faced by the implementers, while exploring areas where design has, and could have been used to solve implementation issues. The conditions discussed will inform user centred design considerations relevant to the design process and raise constraints from real world design implementation for those in need of life altering product design. It will also discuss the design work undertaken for the region of Chikwawa in Malawi for the WATERSPOUTT project, the aim of which has been to produce a combined ceramic filter and SoDis unit for Household Water Treatment and Safe Storage (HWTS) for rural use. SoDis uses a combination of infra-red and UV-A radiation from sunlight to render bacteria and harmful microbes inactive and unable to harm consumers [3]. SoDis typically consists of filling 2L PET water bottles and placing them in direct sunlight for a minimum of 6 hours (longer in cloudy weather conditions). PET bottles allow the radiation to heat the water up to $40-55^{\circ} \mathrm{C}$ for disinfection to take place [4]. It is often the case with aid and intervention projects that an organisation arrives to tackle a specific issue with a prescriptively designed product intended to be the solution of the area's main problem. However, there are many factors that are often neglected including cultural beliefs, local resources and business, and previous intervention projects. Knowledge of these extreme cases of product development and implementation will inform students of some of the factors influencing the acceptability and 
usability of a design. The design process outlined here has been undertaken with the intention of designing a product that can not only be supplied to and adopted by the target users and communities, but also be produced, sold and improved by them. All materials have been selected for their local availability, reusability and compatibility with local craft and manufacturing methods.

\subsection{Design and aid}

More than $\$ 300$ billion has be distributed to Africa in aid spending in the last 50 years, with little improvement in economic or public development. Aid has produced dependant systems where governments can reduce spending on civil development, hampering growth and encouraging corruption [5]. There is a need for investment to help communities become self-sufficient and thrive from their industries. Here, design can be a tool in the production of new products for local sale and growth. This section will discuss the issues surrounding the adoption of HWTS systems, highlighting areas where interventions have been successful and where barriers to adoption have occurred.

The WHO have questioned the reliability of HWTS intervention projects citing multiple studies questioning the reliability of reported results. Suggested factors in the production of inaccurate results have been the 'placebo' effect felt by users, low compliance, limited sample sizes, reporting bias and the limited reductions of exposure to contamination through water alone [6]. The report suggests that HWTS systems are a valuable tool for health and sanitation improvements, though for more reliable results interventions should seek to manage "exposure through multiple pathways" instead of HWTS alone. High levels of compliance should also be ensured with the target communities, as even small amounts of misreporting or a few users continuing to drink small amounts of contaminated water can dramatically affect the final results. This is often the case when users' needs are not being fully met, as well as users succumbing to intervention fatigue, causing participation to wane. In both cases design methods have the potential to improve adoption of the intervention, maintaining performance during testing and post project. For many a product must sell itself, appeal to a user on a basic level and be easily understood without direction. Norman's "Three-level theory" outlines the levels a product must appeal to a user to be perceived positively [7]. On the first level there is a "Visceral" attraction, where the user will be drawn to the aesthetics of the product or designed system. The second "Behavioural" level appeals to the user's ability to use and master the product. The third "Reflective" level is a far deeper connection between the user and the product, appealing to their identity and self-worth. In this third level the user asks if the product reflects an identity that matches them, and if it shows this identity to others. Many interventions tend to focus heavily on the second level, emphasising the function and mastery of a system without exploring the individuals need for identity, self-expression or even a fundamental appreciation of the items aesthetics. Here colour may affect the perceptions of an intervention technology. To improve heat retention in a SoDis container it is sometimes suggested that half of the container be painted black or placed onto a black surface, though culturally this may have different connotations. In the Chewa ethnic group of Malawi black is associated with women and the creation of life, while in a Kenyan study the black colouring of the SoDis bottles were associated with dark magic and clear bottles were preferred [8]. These issues also arise in student projects, where a single feature becomes the priority of the whole product neglecting or compromising other necessary features. Ohrling et al. [9] highlighted this issue and suggested that students needed to constantly consider each features effect on the user, creating ethically viable products for a sustainable future. However, when designing internationally students should not design for their cultural perceptions, instead sustainably catering to the needs and priorities of the user.

Hartinger [10] combined a chimney stove, sink, SoDis and hygiene interventions in their Peru study in an effort to ensure cultural acceptance. Taking a user centred approach, they outlined the importance of creating interventions of culturally acceptable products for implementation, developing systems for local needs and construction methods. The stoves developed were suitable for combating the issues of smoke inhalation and excessive fuel use, as well as being produced from local materials to allow for future large-scale self-dissemination, manufacturing and repair. Multiple designs were offered to discover preferences and efficiency of use. Their attention to users' cultural practices allowed them to observe misinterpretations of the SoDis method with one community placing the bottles in direct moonlight for its "purifying effects" rather than sunlight as intended. Commonly in HWTS interventions the taste of the treated water is contested. Hartinger [10] addressed this issue by performing a blind taste test of treated waters to establish user preference. A year after implementation all chimney stoves and sinks were still in use with users commenting on the improvements the products had made to their lives, 
reducing time required for everyday tasks while simultaneously improving health and wellbeing. Having piped water introduced to the homes, many users chose not to continue with the SoDis, instead choosing to work as a community to chlorinate their reservoir once a month to provide safe water. In this case the task force had attempted to appeal to Norman's three levels of appeal by providing designed, functional and culturally appropriate products. From the Malawi visit there was an understanding that if a previous system or practice worked for a user's ancestors then it must work for them. This was also found by Sanders [11] who found that deeply rooted traditions dictate the physical world of the community. For a community to adopt new technologies the communities must choose to negotiate where tradition and modern tools and practices can co-exist, typically becoming accepted through community discussion or through the approval of local elders of chiefs. The introduction of SoDis to Kathmandu Valley in Nepal [12] is an example of where consideration of local perspectives had not been considered. The community had too little space for the placement of the bottles in the sunlight for long periods, and the treatment of water was considered to make the water less pure. The best solution may have been treating water at source or to develop a system more suitable for the community's lifestyles, beliefs and environment.

Clasen [13] states that analysis of 33 studies found interventions were often effective when combined with other factors such as hygiene or improved water supply. This is not to say that they were more effective than those systems implemented on their own, it is for target users to determine whether to adopt or not. Thus, projects should not only be undertaken with the user's health needs in mind but should be sustainable, affordable, acceptable and scalable within a vulnerable population [13]. Worley [14] echoes this, suggesting that a technology must be understandable, easy to operate and maintain, use locally available resources, be culturally acceptable, affordable, effective and sustainable. This applies to materials and also workers to produce, maintain and operate the technologies. In a later analysis Clasen [15] outlines the importance of adherence to the technology being introduced, finding that a decline from $100 \%$ to $90 \%$ reduces predicted health gains by up to $96 \%$. The largest declines occur when pre-treatment water quality is of higher risk. Further barriers in implementation include affordability and achieving and maintaining supply chains of filtration and chemical resources.

Both studies touched upon product acceptability, from a functional or cultural perspective. Culture adds meaning to a product, attaching ideas, narratives and identity to items [16]. It influences the way we view the world or a person from another part of it. Students should consider their user perceptions when designing products intended for export. For instance, does the user have political or social objections to receiving a product from another country. In Sanders' [11] analysis of tradition and modernity, the two were separate in the minds of the local people. Modernity can be seen as separate from tradition with communities choosing to reject foreign technologies fearing that they threaten to impose onto their locality, impeding traditional practices and forcing them to surrender control over the material and symbolic production and reproduction of their lives. Considering this the aid of an NGO or government body can be vital in communicating a product to the target community, though this can be problematic where long-term sustainability is concerned as NGOs typically depend on external funding [17]. Products must therefore be designed for adoption by the community so as to allow them to produce and maintain the systems themselves. Traditionally students are taught to design for consumers in the developed world, forgetting the needs of wider cultures and beliefs. In Louden [18] Welsh students were taken to Ahmedabad, India for a collaborative project with local students. Attempting to develop sustainable water solutions for users in both countries, students were able to gain a general understanding of local culture within a short period of time. On return to the UK they were asked to design a product for both cultures without direct Indian involvement. It was raised that the students would have benefited from continued feedback from the Indian students during the design phase and more tightly managed deliverable requirements. Though the students practiced a suitable product development process, the intention of designing for multiple cultures at once, and the lack of co-design from their Indian partners the final designs were not suitable for their intended markets. Projects like these encourage students to look outside of their own personal cultural context and engage more thoroughly with their ethnographic research practices, studying and learning from systems cultures develop to suit a local context. To gain access to these solutions students must engage in co-design with project partners or designers in that context. Students may also be a valuable resource for concept generation and research by involving them in an intervention project in a secondary role supporting a project investigator. Here they can contribute to a project and develop their skills whilst maintaining realistic deadlines within the context of the student experience. 


\subsection{Development of a combined filter and SoDis system.}

WATERSPOUTT aimed to design and implement a combined solar ceramic HWTS for Chikwawa, Malawi. Adhering to criteria suggested by Clasen [13] and Worley [14] the aim was to produce a water purification system that is culturally acceptable, produced using local crafts and resources, has minimal environmental impact and can be produced by the user. These steps provide opportunities for income generation and local development, while avoiding barriers of affordability and achieving and maintaining supply chains with foreign suppliers. Four major designs were produced and tested for their appeal to the local communities, their effects on bacteria and turbidity and ability to be understood and used. The first unit produced consisted of a two-stage ceramic filter with non-permeable walls for use with a locally manufactured clear polypropylene bucket (figures 1-2).
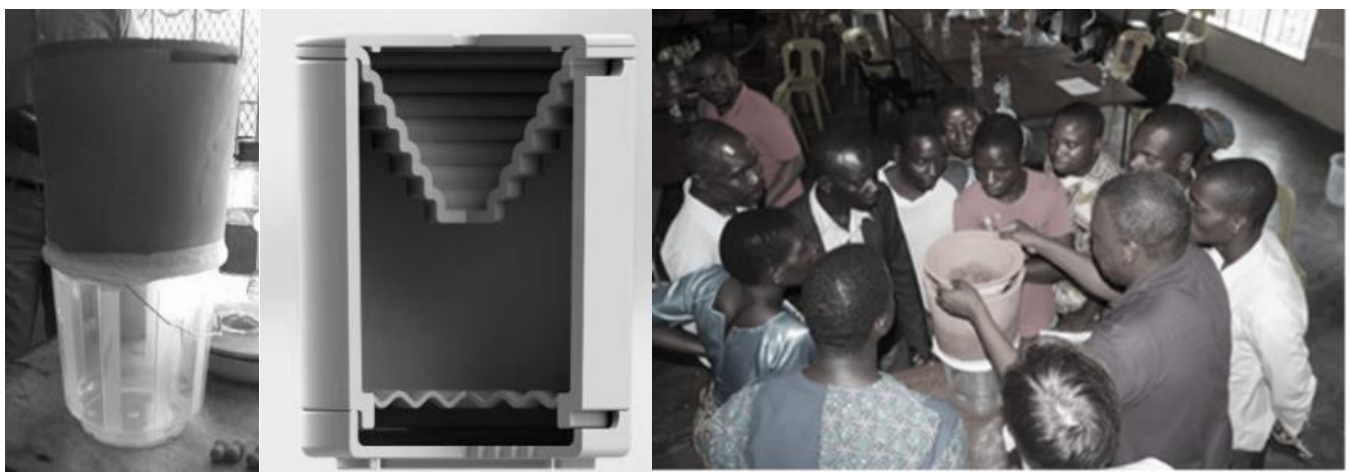

Figure 1-3. 1: First ceramic filter as displayed in Nchalo, Chikwawa shared dialogue workshop. 2: Cut away view of two stage ceramic filter shown in in fig 1. 3: Community representatives observe and comment on the filter design at Nchalo workshop

Ceramic filtration reduced turbidity of the local water allowing for more efficient disinfection through SoDis. In May 2017 the researchers attended a shared dialogue workshop in Nchalo organised by WATERSPOUTT partners at the University of Malawi (UNIMA). Community representatives were asked to give their feedback on the design. They commented that the product was aesthetically pleasing, and that they were particularly happy that the design could be made, sold and repaired by them, removing their dependence on others. Constructively, the group requested a faster filtration time (20 litres in $<2$ hours), that handles be added to ease handling the filter, and for multiple styles to be produced to cater for different tastes. Taking these comments into consideration further testing was undertaken with different mixtures of sawdust and clay. It was found that that the more porous the clay became the more fragile it would become. Even at its most porous the filters were never able to drop below the twohour time requirement prompting the creation of two designs, the pot media filter and the pillow filter. The pot filter consisted of a pot with an open base with a fabric membrane at its base and a fabric bag placed at its top. Each fabric barrier held porous ceramic media that effectively reduced the turbidity of the of the water being filtered. The second design consisted of a fabric pillow filled with the same ceramic media designed to be placed over a hole at the base of a bucket.
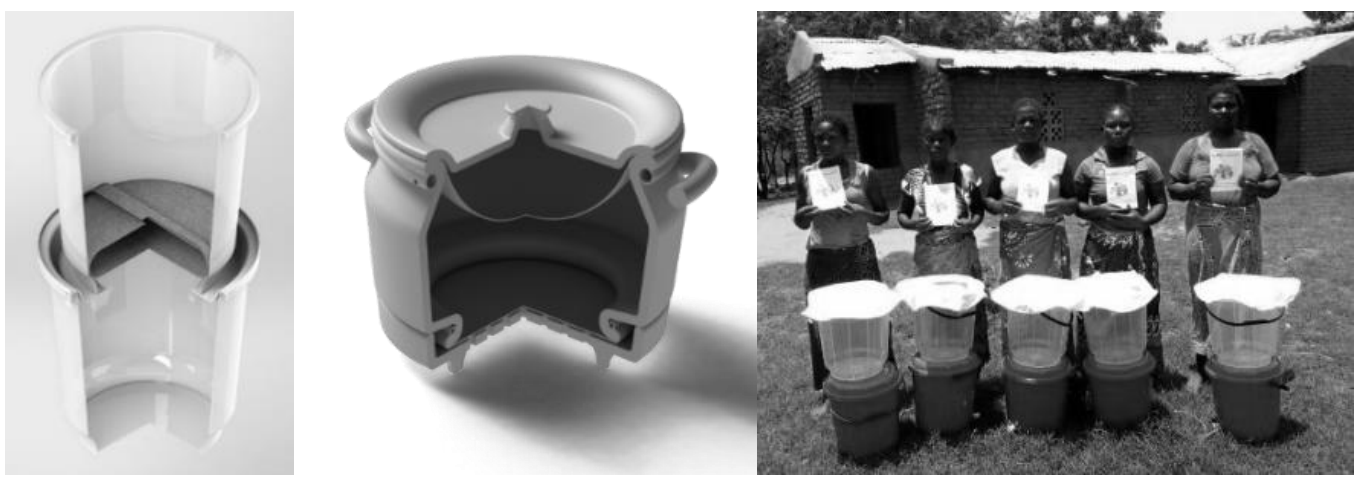

Figure 4-6. 4: Cut away view of the pillow filter design. 5: Cut away view of the pot filter design. 6: Chikwawan women receive SoDis buckets and fabric filters for initial testing 
Tests at CIEMAT-Platforma Solar de Almeria, Spain showed that the pot filter reduced the turbidity by $86-96 \%$ and the pillow filter showing turbidity reductions of $30 \%$. The pot filter was selected for initial user testing in Chikwawa. Prototypes were produced at nearby Dedza pottery however due to high costs the units were not carried forward to the next project stage. Due to time constraints further development of the ceramic filter was not possible leading to the production of a fabric filter. During initial testing of the fabric filter units both in Chigweshe and at UNIMA there was an average turbidity reduction of $60 \%$. Unlike the pot filters which required an hour and a quarter to filter 20L, only ten minutes was needed using the fabric filter. It is noteworthy that the reduced time does show a significant reduction in quality of the filtration taking place. This is largely as a result of fewer filter stages and larger pour sizes being used in the fabric filter.

\section{DISCUSSIONS \& CONCLUSIONS}

This study has found that as a designer, one may be predisposed to discover a solution for a need and then find the correct processes and materials required for making that solution into a physical product. Instead the constraints of an area and culture require a designer to seek a solution through the rigid adherence of a fixed selection of materials and processes, creating sustainable products intended for development, not obsolescence and replacement. This approach will aid students in their production of products for the real world, encouraging them to maximise the potential of each part and using locally sustainable materials rather than relying on specially treated parts from a complex supply chain. The development of the combined filtration and SoDis unit relied heavily on the primary and secondary cultural research undertaken.

The implementation of any form of development product should always include a level of co-design with the local community adding greater context to the design produced, as shown by Hartinger [9] and Louden [18]. User considerations were the leading motivator for innovation during the design process. It is recommended that students be introduced to these extreme design scenarios to enable them to consider the wider effects of their design choices while working to strict regional constraints. Although the product was designed to meet a specific need, it cannot be ignored that its usability, time demands, aesthetics and manufacturability are factors that transcend the immediate need for potable water and affect other aspects of daily needs. The users had concerns about product time requirements with many of them already having many demands on their time.

As shown by Louden [18] designing at a distance without regional representation can leave designers producing outcomes that do not meet user requirements. Women are traditionally responsible for the collection and supply of water to families, and in areas with a harsh climate it would be unreasonable to expect this to be their only role. Often acting as farmers, selling crops, caring for families, preparing meals and performing other traditional and social roles, time is a precious commodity. In many aid and intervention projects that the target user is a real person with all the wants, needs and desires of any other can easily be forgotten.

Designers and especially design students should remember that user requirements transcend class, culture and lifestyle. No one wants inelegant objects in their house, especially not objects that do not take into consideration their fundamental cultural and independent beliefs or aspirations. Simplified examples of user considerations like Normans' three level theory provide students with a reminder that user needs extend beyond a singular issue and require designers to provide meaningful and personal solutions. Design students can become attached to a specific perception of design that does not transcend the realms of their own culture or available resources. Students should be aware of the wider design world and the contributions they are able to make to disciplines outside of their own. This would encourage a desire to design for other, different cultures and improve their employability in a wider network of roles in an increasingly globalised society, where the future of trade and global value chains are reshaping industry and commerce. 


\section{REFERENCES}

[1] Papanek V. Design for the Real World, 1971 (Academy Chicago Publishers, Chicago).

[2] WHO. Drinking water. Available: https://www.who.int/en/news-room/factsheets/detail/drinking-water [Accessed 2019, 18/1/19] (2018) 7 February.

[3] Wegelin M. Sommer B. Solar Water Disinfection (SoDis) - Destined for Worldwide Use? Waterlines, Volume 16, 1998, pp30-32.

[4] McGuigan K.G., Joyce T.M. and Conroy R.M. Solar Disinfection: Use of Sunlight to Decontaminate Drinking Water in Developing Countries. Journal of Medical Microbiology, Volume 48, 1999 pp.785

[5] Moyo D. Dead Aid, Why Aid is not working and how there is another way for Africa, 2009 (Penguin Books, London).

[6] WHO. Considerations for policy development and scaling-up household water treatment and safe storage with communicable disease prevention efforts. World Health Organisation 2012, pp.1215.

[7] Berger W. Glimmer: How design can transform your business, your life and maybe even the world, 2009 (Random house group Ltd., London).

[8] Burgess S. and Collins O. Solar disinfection of water - a case study from Kenya, Waterlines, Volume 22. No.4, 2004.

[9] Ohrling T., Normark J. and Wikberg Nilsson A. Norm Creativity in student design projects-one approach to creating sustainable societies. In Int. Conference on Engineering and Product Design Education 2018. P344-349.

[10] Hartinger S.M., Lanata C.F., Gil A.I. and Hattendorf J. Combining interventions: improved chimney stoves. Kitchen sinks and solar disinfection of drinking water and kitchen cloths to improve home hygiene in rural Peru. Facts Reports, 2012.

[11] Sanders T. Reconstructing witchcraft: Postcolonial Africa and analytic (Un) certainties. American anthropologist, 2003, pp.338-348.

[12] Rainey R.C. and Harding A.K. Acceptability of solar disinfection of drinking water treatment in Kathmandu Valley, Nepal. International Journal of Environmental Health Research, 2005 pp. 361-372.

[13] Clasen T., Schmidt W.P., Rabie T., Roberts I. and Cairncross S. Interventions to improve water quality for preventing diarrhoea: systemic review and meta-analysis. BMJ, 2007.

[14] Worley M.T. Technology for community development in central Asia. Joint Development Associates. 2005 pp. 27-28.

[15] Clasen T. Household water treatment and safe storage to prevent diarrheal disease in developing countries. Current environmental health reports, Springer, 2015, pp. 69-73.

[16] Barnwell M. Design, Creativity and Culture: An orientation to design. 2011 (Black Dog Publishing, London).

[17] Gurung P., Grimm B. and Autenrieth M. Disseminating the SoDis method: which approach is most effective? Waterlines, Volume 28, 2009, pp.136-137.

[18] Louden G., Watkins C., D'Onofrio A., Hopkins H. and Ancelot E. Collaborative research model for designing sustainable water usage solutions. Research into Design for Communities, Volume 1. ICoRD 2017. Smart Innovation, Systems and Technologies, Vol 65. 2017.

The WATERSPOUTT project described in this paper has received funding from the European Union's Horizon 2020 research and innovation programme under grant agreement number 688928 